\section{Protocolo abreviado de resonancia magnética en espondiloartritis: más allá de la sacroileítis}

\author{
GIANCARLO SCHIAPPACASSE F. ${ }^{1}$, JORGE DÍAZ J. ${ }^{1}$, \\ PABLO ALVAYAY Q. ${ }^{2, a}$.
}

\section{A brief magnetic resonance imaging protocol for spondyloarthritis}

\begin{abstract}
Spondyloarthritis is a group of diseases that seriously hampers quality of life. Diagnostic criteria, in which images play an important role, have evolved over time. The most recent diagnostic criteria, published in 2009, included magnetic resonance imaging (MRI) of the sacroiliac joints for the first time. This technique achieves an early diagnosis and provides a useful tool for the evaluation and prediction of response to biological therapy. Herein, we describe the different MRI findings in spondyloarthropathies. We also highlight the use of a protocol that includes the sacroiliac joints and spine and that does not consider the routine use of paramagnetic contrast.
\end{abstract}

(Rev Med Chile 2015; 143: 905-912)

Key words: Magnetic Resonance Imaging; Sacroiliitis; Spondylarthritis, ankylosing; Spondylitis, Ankylosing.

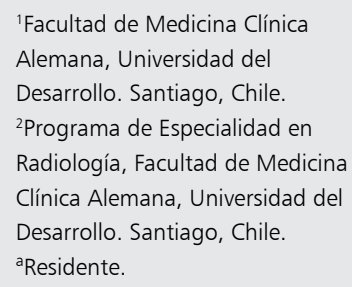

Recibido el 20 de septiembre de 2014, aceptado el 26 de abril de 2015.

Correspondencia a:

Dr. Giancarlo Schiappacasse F gschiappacasse@alemana.cl
I a espondiloartritis (SA) corresponde a un grupo de enfermedades reumatológicas con características en común. Estas incluyen a la espondilitis anquilosante (EA), artritis reactiva, antiguamente conocida como síndrome de Reiter (AR), artritis psoriática (AP), artritis asociada a enfermedad inflamatoria intestinal y la espondiloartritis indiferenciada. La asociación con el antígeno leucocitario humano B27 (HLA-B27) y una serie de manifestaciones clínicas axiales y periféricas unen a estas entidades diagnósticas.

Los criterios diagnósticos han evolucionado en el tiempo, es así como los criterios de Roma ${ }^{1}$ que se desarrollaron en el año 1961, de New York modificados del año $1984^{2}$ y los de Amor del año $1990^{3}$ consideran los hallazgos radiográficos como criterio diagnóstico. Sin embargo, estos últimos no permiten una detección precoz de la enfermedad, retrasando el diagnóstico de 6 a 8 años desde el inicio de los síntomas ${ }^{4}$. La resonancia magnética (RM), a partir de la década 1990-99, ha surgido como una herramienta atractiva para la detección precoz, adelantando el diagnóstico de la enfermedad varios años si se compara con la radiografía ${ }^{5}$. El grupo The Assessment of SpondyloArthritis international Society (ASAS) desarrolló en el año 2009 los criterios que consideran por primera vez a la RM como herramienta fundamental en el diagnóstico de la sacroileítis, no incluyendo otros fenómenos del esqueleto axial ${ }^{6}$.

El propósito de este trabajo es remarcar el rol de la RM en el diagnóstico precoz de las SA y difundir un protocolo de RM que hace énfasis en los hallazgos axiales a nivel de la columna vertebral y en articulaciones sacroilíacas.

\section{Rol de la RM en las espondiloartritis}

Los criterios ASAS, publicados el año 2009, fueron los primeros en considerar los hallazgos de sacroileítis en RM como parte de uno de sus brazos diagnósticos. Hay que considerar que estos se aplican a pacientes menores de 45 años con 
dolor lumbar de tres o más meses de duración. Así, se desarrollan dos brazos diagnósticos: uno radiológico y otro clínico. El criterio radiológico se basa en la demostración de sacroileítis mediante radiografía simple según los criterios de New York de $1966^{7}$ o a través de RM.

En la última década se han visto importantes avances en el tratamiento precoz de las SA, gracias al desarrollo de terapias biológicas como son los antagonistas del factor de necrosis tumoral- $\alpha$ (Anti TNF- $\alpha$ ), de ahí la necesidad de incluir a la RM para el diagnóstico precoz de estas entidades. La RM permite hacer el diagnóstico al menos 3 años antes, si se compara con el estudio radiológico simple ${ }^{5}$. Es importante mencionar que sólo los signos de inflamación activa y no las alteraciones estructurales son válidas para el diagnóstico e inicio de terapia biológica.

En pacientes que ya están en tratamiento con terapia biológica, la RM permite evaluar el grado de actividad en el esqueleto axial, monitorizando la evolución de la enfermedad ${ }^{8-10}$. De esta misma forma, se ha visto que bajo ciertas condiciones se puede predecir la respuesta al tratamiento ${ }^{11}$.

\section{Evaluación actual de la espondiloartritis con RM}

En nuestro servicio de imagenología se ha implementado un protocolo para la evaluación de espondiloartritis que se realiza desde octubre del año 2012, basado en protocolos sugeridos internacionalmente. Las secuencias empleadas se describen en la Tabla 1 y Figura 1.

No sólo se deben evaluar las articulaciones sacroilíacas buscando hallazgos que cumplan con los

Tabla 1. Protocolo de RM en espondiloartritis

\begin{tabular}{|l|}
\hline Columna total (C-D y D-L) \\
T1 SE Sagital \\
STIR Sagital \\
Articulaciones sacroilíacas \\
T1 SE Coronal \\
STIR Coronal \\
T1 fat-sat Coronal \\
\hline
\end{tabular}

C-D: Cérvico-dorsal; D-L: Dorso-lumbar. SE: Spin-Echo. STIR: Short Tau Inversion Recovery. Fat-sat: Saturación de la grasa.

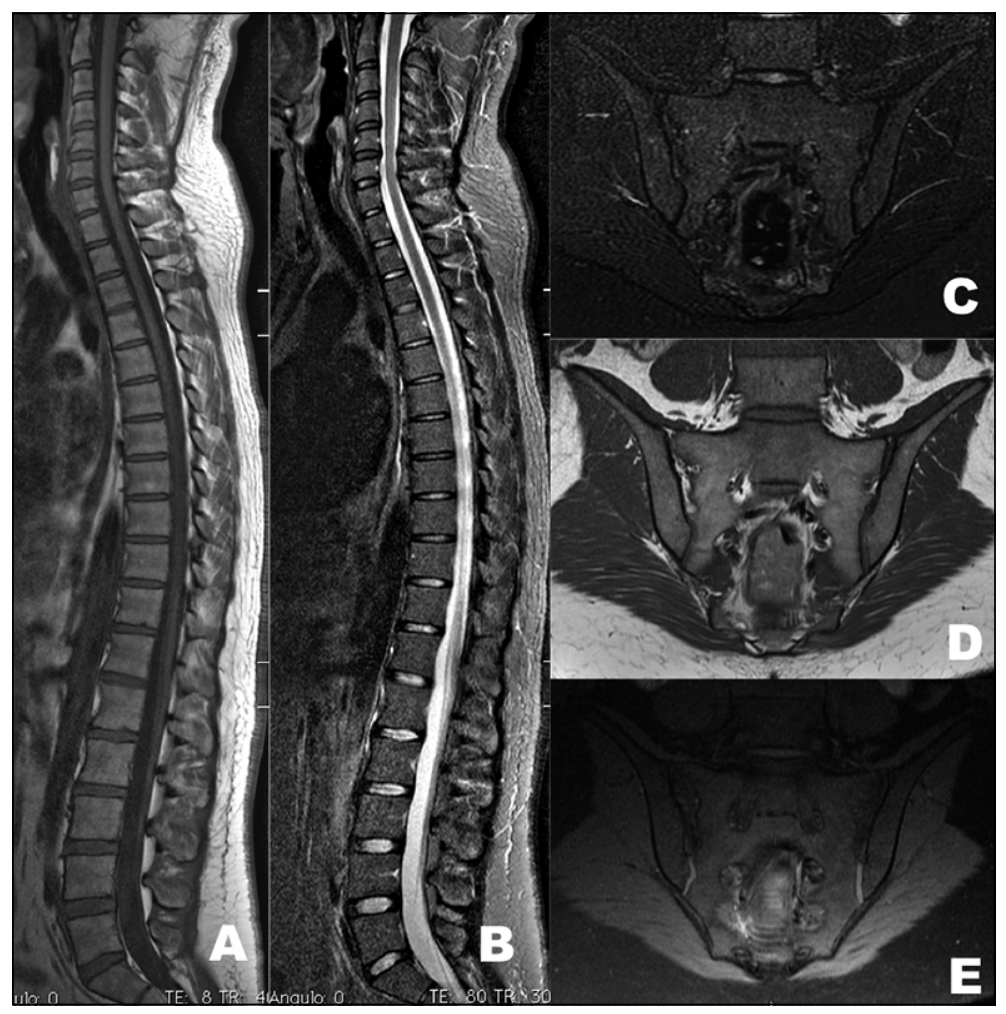

$\mathrm{B}$
Figura 1. Imágenes de resonancia magnética del protocolo de espondiloartritis en un paciente sin hallazgos patológicos. Corte sagital de columna total en A) T1 SE y B) STIR. Corte coronal de articulaciones sacroilíacas en C) STIR; D) T1 SE y E) T1 fat sat. 
criterios ASAS de SA, también se debe estudiar la columna vertebral, dado que hasta $23 \%$ de los pacientes con enfermedad clínicamente activa tiene lesiones vertebrales sin alteración en las articulaciones sacroilíacas (Figura 2) ${ }^{11}$. En esta localización el segmento más afectado es el dorsal ${ }^{12}$. Como se menciona más adelante, la carga inflamatoria de la columna vertebral permite además estimar la respuesta a la terapia con Anti TNF- $\alpha^{11}$.

El protocolo contempla la evaluación de la columna vertebral en 2 bloques: uno cérvico-dorsal y otro dorso-lumbar, que luego son unidos en una sola imagen para favorecer la interpretación. Se prefiere la realización de secuencias sagitales T1 spin-echo (SE) y Short Tau Inversion Recovery (STIR) (Figuras 1A y 1B). Las secuencias T1 SE permiten la detección de lesiones estructurales, entre ellas erosiones. La secuencia STIR permite detectar lesiones agudas que podrán ser detectadas al eliminar la alta señal del tejido adiposo adyacente a las lesiones.

Las imágenes de las articulaciones sacroilíacas se basan en secuencias coronales STIR, T1 SE y T1 con saturación de la grasa (fat-sat) (Figuras 1C, $1 \mathrm{D}$ y $1 \mathrm{E}$ ). Estas secuencias coronales se realizan en paralelo a la línea que pasa por el margen superior y dorsal de S1 y S3.

Las secuencia STIR en las articulaciones sacroilíacas permiten detectar lesiones inflamatorias, mientras las secuencias T1 y $\mathrm{T} 1$ fat-sat son útiles en la evaluación de lesiones estructurales.
Existe controversia sobre la realización de secuencias STIR y la necesidad de realizar secuencias T1 fat-sat con gadolinio (T1/Gd) para evaluar la columna vertebral y las articulaciones sacroilíacas. Ambas son útiles en la detección de lesiones inflamatorias agudas (edema de médula ósea y osteítis). La secuencia STIR muestra áreas de alta señal basándose en el tiempo de relajación T2 del agua libre, mientras la secuencia T1/Gd muestra áreas de alta señal debido al aumento de la vascularización. En nuestro centro no se realiza de rutina imágenes $\mathrm{T} 1 / \mathrm{Gd}$, evitando posibles complicaciones en pacientes con insuficiencia renal o alergia al contraste paramagnético, además, disminuir el tiempo de adquisición del estudio. Existen estudios que concluyen que la secuencia STIR es comparable a las secuencias contrastadas para detectar inflamación periarticular sacroilíaca ${ }^{13} \mathrm{y}$ en estudio de columna vertebral ${ }^{14}$.

\section{Hallazgos en RM: lesiones inflamatorias (agu- das) y estructurales}

Según el protocolo de RM que hemos utilizado en nuestro servicio de imagenología para evaluar SA, se pueden identificar lesiones inflamatorias agudas y lesiones estructurales o crónicas. En las articulaciones sacroilíacas y columna vertebral se pueden identificar las lesiones listadas en la Tabla 2.

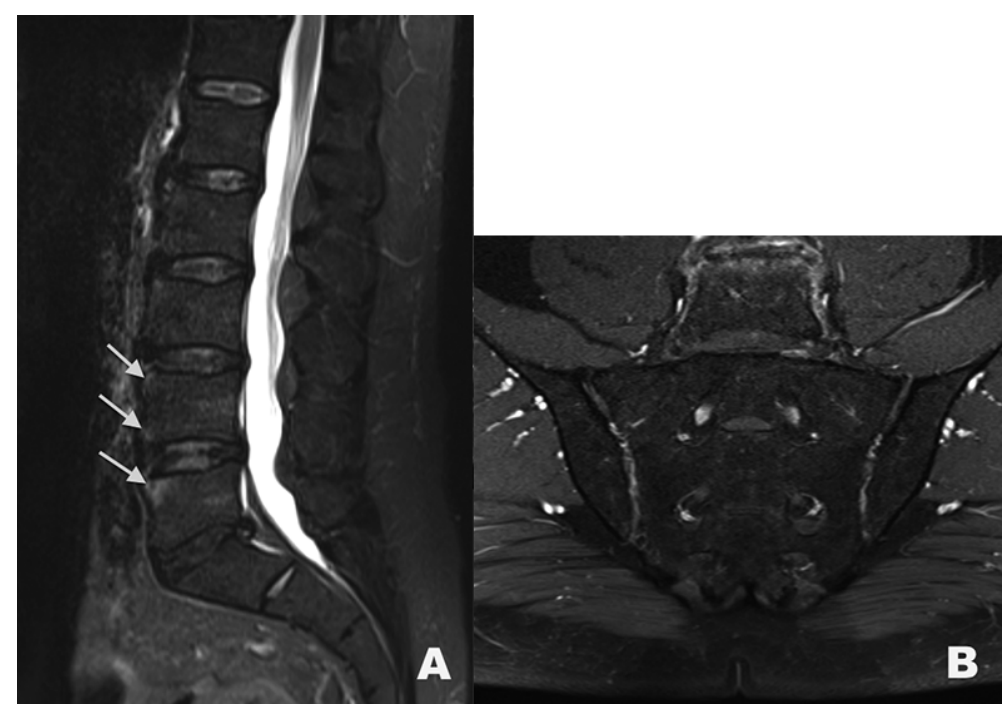

Figura 2. Imágenes de RM, secuencia STIR, de un paciente con compromiso de vertebras lumbares, sin alteración de articulaciones sacroilíacas. Nótese el aumento de señal de los vértices vertebrales anteriores de las vertebras L4 y L5 en A). 
Tabla 2. Hallazgos en RM en espondiloartritis

\begin{tabular}{|lc|}
\hline Articulaciones sacroilíacas & Columna vertebral \\
\hline Lesiones inflamatorias agudas & Lesiones inflamatorias agudas \\
Edema de médula ósea y osteítis & Espondilitis (Lesión de Romanus) \\
Sinovitis & Espondilodiscitis \\
Capsulitis & Entesitis de ligamentos espinales \\
Entesitis & \\
Lesiones estructurales & Lesiones estructurales \\
Erosiones & Depósitos de grasa en los vértices vertebrales \\
Depósitos de médula ósea grasa periarticular & Sindesmofitos y espolones \\
Puentes óseos y anquilosis & Calcificación discal \\
\hline
\end{tabular}

Lesiones inflamatorias agudas de las articulaciones sacroilíacas

\section{Edema de médula ósea y osteítis}

El edema de médula ósea se presenta en hasta 90\% de las SA (Figura 3A), pero también se puede ver en otras condiciones, como en enfermedades degenerativas y entre 2,6-20\% de los sujetos sanos $^{15}$. En RM se manifiesta como zonas de hiperintensidad en secuencia STIR e hipointensidad en secuencias ponderadas en T1. La señal de referencia está dada por la señal de la médula ósea de la zona interforaminal del sacro. La osteítis se diagnostica en secuencias T1/Gd cuando se produce impregnación de algunas zonas por aumento de la vascularización y perfusión, reactiva a la inflamación ${ }^{16}$.

El diagnóstico de sacroileítis en RM según criterios ASAS se establece cuando existe edema de médula ósea y/u osteítis en situación periarticular o subcondral. Cuando sólo existe un foco de señal alta se necesita su presencia en a lo menos 2 cortes consecutivos. Si existe más de un foco de señal alta, puede ser suficiente con 1 corte para el diagnóstico.

\section{Sinovitis}

Se reconoce como zonas de aumento de señal en el margen sinovial del espacio articular en secuencias T1/Gd. En secuencia STIR no se logra distinguir del líquido sinovial ${ }^{16}$. Es muy inusual su presentación aislada (sin edema de médula ósea) y no constituye un criterio diagnóstico.

\section{Capsulitis}

Similar a la sinovitis, pero el aumento de señal se observa en la cápsula anterior y posterior. Se puede extender medial o lateralmente en el periostio $^{16}$.

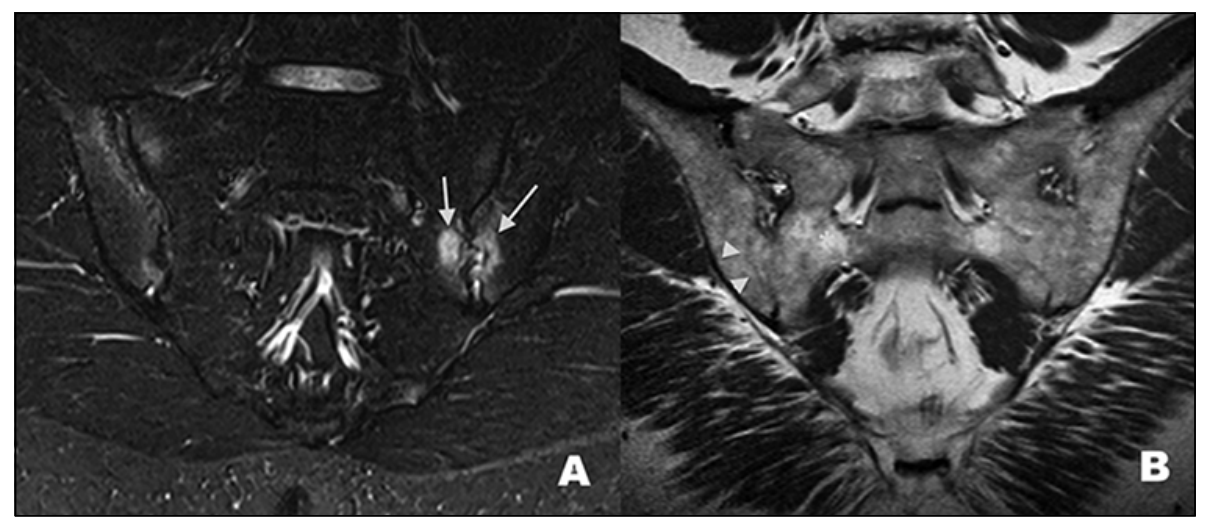

Figura 3. Lesiones inflamatorias agudas y estructurales de las articulaciones sacroilíacas. A) Corte coronal de RM en secuencia STIR que muestra áreas de aumento de la señal compatibles con focos de edema de médula ósea subcondral (fechas); B) Corte coronal de RM en secuencia T1 que muestra pérdida de la continuidad de la superficie articular compatible con puente óseo (cabeza de flecha) y áreas de aumento de señal de la médula ósea compatible con depósito de tejido adiposo (asterisco). 


\section{Entesitis}

Aumento de señal en el sitio de inserción de tendones y ligamentos en secuencias STIR y T1/ Gd. Se puede asociar a edema de medula ósea y de partes blandas adyacentes ${ }^{16}$.

\section{Lesiones estructurales de las articulaciones sacroilíacas}

\section{Esclerosis subcondral}

Áreas hipointensas en todas las secuencias de RM. No se impregnan con el uso contraste. Deben tener un grosor mínimo de $5 \mathrm{~mm}$ desde el espacio articular sacroilíaco, para no confundirlas con áreas de esclerosis de pacientes normales ${ }^{16}$.

\section{Erosiones}

Son zonas de baja señal en T1 y que pueden tener señal alta en STIR si están activas. La secuencia T1 fat-sat puede ser la más útil para detectarlas. Se observan como áreas donde existe pérdida de la definición de la línea cortical que inicialmente se pueden ver aisladas, habitualmente en el lado iliaco de la articulación y que, en la medida que confluyen, pueden afectar a ambas superficies articulares y dar una apariencia de pseudo-ensanchamiento a la articulación ${ }^{16}$.

\section{Depósito de médula ósea grasa periarticular}

Áreas de mayor señal en secuencias ponderadas en T1 (Figura 3B). No es un hallazgo específico y probablemente representan zonas de inflamación $\operatorname{reparada}^{16}$.

\section{Puentes óseos y anquilosis}

Se observan como áreas de baja señal en todas las secuencias y pueden estar rodeadas por zonas de alta señal en secuencias ponderadas en $\mathrm{T} 1$ que representan depósitos de grasa ${ }^{16}$. En estados avanzados generan pérdida de la definición del espacio articular y fusión ósea (Figura 3B).

\section{Lesiones inflamatorias agudas de la columna vertebral}

\section{Espondilitis (Lesión de Romanus)}

Inflamación en el sitio de inserción del ligamento longitudinal anterior a los cuerpos vertebrales y discos (Figura 2A). Da inicio a las erosiones en los márgenes anteriores disco-vertebrales. Las lesiones tienden a curar por proliferación de hueso esclerótico produciendo encuadre del cuerpo vertebral, debido a la pérdida de la concavidad normal de la superficie vertebral anterior. $\mathrm{La}$ apariencia típica es de áreas de baja intensidad de señal en secuencias ponderadas en T1 y marcada hiperintensidad de señal en secuencias STIR y T1/ $\mathrm{Gd}^{17}$. Estas lesiones se observan hasta en $67 \%$ de los pacientes con SA. Cuando hay más de 3 lesiones en ausencia de nódulos de Schmorl, su especificidad es de $81 \%$ y puede subir hasta $97 \%$ en pacientes menores de 40 años ${ }^{18}$.

Las lesiones de Romanus, cuando se encuentran en número $\geq 3$ o son severas en sujetos menores de 50 años, al igual que lesiones leves a moderadas de elementos posteriores en secuencias de RM con saturación de la grasa, presentan valores pre-test muy altos (likelihood ratios $>10$ ) según un estudio realizado por Bennett et $\mathrm{al}^{18}$, donde demostró que en columna pueden ser altamente específicas de SA, incluso sin conocer la historia clínica del paciente.

\section{Espondilodiscitis}

Las erosiones y destrucción disco-vertebrales encontradas en EA fueron originalmente descritas por Andersson en el año 1937. Se pueden observar hasta en $33 \%$ de los pacientes con $\mathrm{SA}^{18}$. Han sido clasificadas en 3 tipos. La lesión tipo 1 compromete la región central de la unión disco-vertebral, está cubierta por la plataforma vertebral y tiene la misma apariencia de un nódulo de Schmorl. La RM muestra destrucción de la plataforma asociada a herniación discal intravertebral y cambios inflamatorios. Después de la inyección de gadolinio se observa aumento de la señal de la médula ósea. La lesión tipo 2 compromete la porción periférica de la unión disco-vertebral, no cubierta por plataforma cartilaginosa. La lesión tipo 3 compromete la porción central y periférica de la unión disco-vertebral y se observa en etapas avanzadas de la enfermedad. La RM va a demostrar mayor destrucción de la plataforma vertebral, edema de médula ósea, cambios adiposos o esclerosis ${ }^{17}$.

\section{Lesiones de las articulaciones facetarias, costo-vertebrales y costo-transversas}

Inicialmente se observa edema de médula ósea y el compromiso inflamatorio de los tejidos blandos adyacentes (Figura 4A). Posteriormente se hacen evidentes las erosiones y esclerosis, que puede fina- 


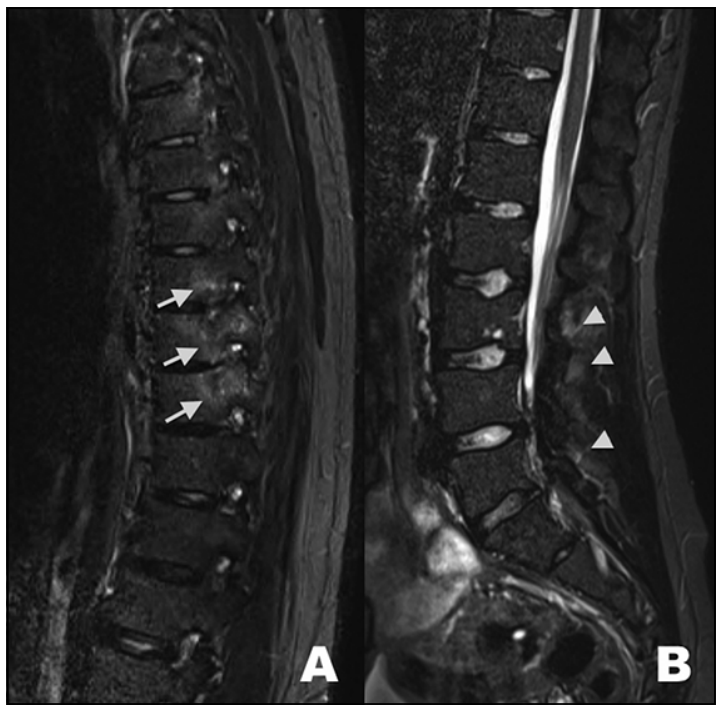

Figura 4. Lesiones inflamatorias agudas de columna vertebral en secuencia STIR. A) Corte sagital del segmento torácico que muestra áreas de aumento de señal en relación a las articulaciones costovertebrales (flechas); B) Corte sagital del segmento lumbar que muestra áreas de aumento de señal en el sitio de inserción de ligamentos espinales posteriores, compatibles con focos de entesitis (cabezas de flechas)

lizar con la anquilosis articular, cuya manifestación consiste en la rectificación de la lordosis lumbar y cervical y exageración de la cifosis dorsal.

El compromiso inflamatorio cuya evolución en el tiempo lleva a la anquilosis de las articulaciones costo-transversas puede causar reducción de la expansión torácica. La osificación de la cápsula articular interapofisiaria da lugar a líneas verticales densas que, junto a la osificación de los ligamentos supra e interespinosos en radiografía frontal, da la apariencia de "línea de trolebús".

\section{Entesitis de ligamentos espinales}

La entesitis de ligamentos espinales (Figura 4B) se caracteriza por aumento de la señal en el sitio de inserción de los ligamentos supraespinoso, infraespinoso y ligamento amarillo en secuencias STIR y T1/Gd. Este hallazgo tiene una especificidad de $87 \%$ para $\mathrm{SA}^{18}$.

\section{Lesiones estructurales de la columna vertebral}

Depósitos de grasa en los vértices vertebrales

Los depósitos de grasa en el hueso son preferentemente visualizados en secuencias ponderadas

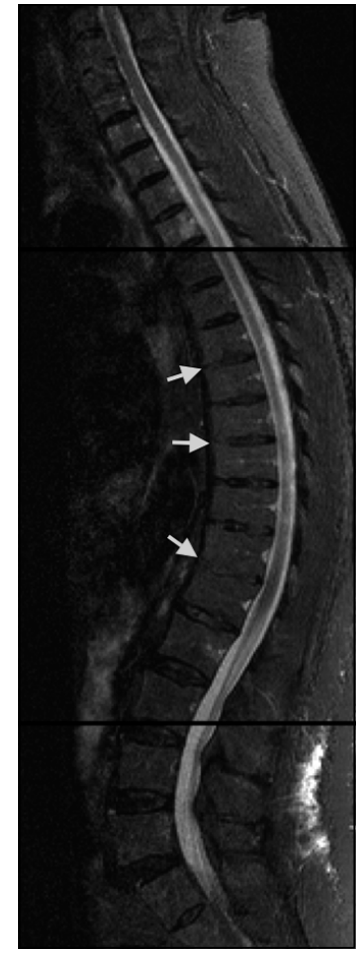

Figura 5. Corte sagital de la columna vertebral en secuencia STIR que muestra sindesmofitos anteriores (flechas) del segmento toracolumbar asociado a encuadre vertebral.

en T1. Como se mencionó previamente, éstos indican áreas de inflamación previa y en la actualidad se considera que son predictivas de la formación de sindesmofitos. Algunos estudios sugieren que la presencia de más de 5 de estas lesiones son altamente sugerentes de una SA $(\mathrm{LR}=12,6)^{19}$.

\section{Sindesmofitos y espolones}

Los sindesmofitos corresponden a la osificación del anillo fibroso y se visualizan como proyecciones óseas finas orientadas verticalmente ${ }^{17}$ (Figura 5), algunas veces difíciles de ver. Predominan en las superficies anteriores y laterales de la columna vertebral y se pueden expandir eventualmente al espacio discal. La osificación puede extenderse al ligamento longitudinal anterior y tejidos paraespinales y esto da una apariencia conocida como columna en "caña de bambú". La RM muestra engrosamiento y baja señal de los ligamentos longitudinal anterior y posterior en adición a la fusión de cuerpos vertebrales. Los discos intervertebrales suelen mantener su altura.

\section{Calcificación discal}

En pacientes con SA es común observar cal- 
cificación de distintas porciones del disco intervertebral. Se han descrito 4 patrones diferentes: marginal, anular, nuclear y sólido. Las áreas calcificadas se reconocen como zonas de hiperintensidad de señal en secuencias ponderadas en T1. Se piensa que esto se debe a la estructura física de los depósitos de calcio ${ }^{17}$.

La actividad inflamatoria de la columna vertebral y de las articulaciones sacroilíacas puede ser sometida a métodos de puntuación con RM. En las articulaciones sacroilíacas, el método que ha demostrado mejor reproducibilidad interobservador y sensibilidad a los cambios es el desarrollado por The Spondyloarthritis Research Consortium of Canada (SPARCC) ${ }^{8,9}$. En la columna vertebral se han desarrollado varios sistemas con adecuada reproducibilidad y sensibilidad a los cambios, entre ellos The Ankylosing Spondylitis Spine Magnetic Resonance Imaging Activity Score (ASspiMRI) y su modificación simplificada de Berlín, el score Leeds y SPARCC ${ }^{10}$. Aunque los sistemas de evaluación de la actividad inflamatoria no se han vuelto muy populares en la práctica clínica, estos han encontrado su espacio en el área de la investigación y desarrollo de ensayos clínicos.

La RM de la columna vertebral tiene un rol en la predicción de la respuesta a la terapia biológica. En el año 2008, Rudweilt et al ${ }^{11}$ mostraron que en el contexto de una enfermedad de corta duración (menor a 10 años) y proteína C reactiva elevada, una carga inflamatoria alta en la RM de columna vertebral (score de Berlín $\geq 11$ ) podía predecir que pacientes responderían a la terapia biológica.

El pronóstico a largo plazo de la enfermedad está dado por la formación de sindesmofitos y fusión vertebral. Sin embargo, hay pacientes que no evolucionarán a esta fase. Los estudios de RM han mostrado que los sindesmofitos se pueden desarrollar desde lesiones inflamatorias agudas tipo osteítis/entesitis ${ }^{20} \mathrm{o}$ desde infiltraciones adiposas $^{21}$, incluso en pacientes que recibieron terapia Anti-TNF- $\alpha$.

\section{Discusión}

Los beneficios de aplicar un protocolo de RM con la tecnología disponible permite optimizar los tiempos de estudios, evitar el uso de contraste paramagnético y disminuir las molestias de los pacientes que se ven sometidos a este tipo de estudio.
La RM, en la última década, ha demostrado ser útil en la evaluación de pacientes con espondiloartritis, ya sea en su diagnóstico precoz, control del tratamiento con terapia biológica, evaluación de la actividad inflamatoria como también en la determinación del pronóstico en pacientes seleccionados.

Existen variados métodos que utilizando la RM estiman el grado de inflamación de las articulaciones sacroilíacas y columna vertebral, y aunque estos no son populares, pensamos que en un futuro cercano estos sistemas formarán parte de la práctica diaria del reumatólogo y radiólogo, en la medida que se vuelva común el control de los pacientes con SA y terapia biológica.

Pese a que la inclusión de la RM ha sido importante en el diagnóstico y tratamiento con terapia biológica en fases temprana de la enfermedad, pensamos que se debe ser cauto y que desde el punto de vista del radiólogo los informes deben concluir los hallazgos como "altamente sugerentes de sacroileítis" por la posibilidad de falsos positivos y considerando que estos son un criterio importante, pero no absoluto, para establecer el diagnóstico de una espondiloartritis.

Se han descritos algunos hallazgos a nivel de la columna vertebral que son altamente específicos de espondiloartitris y, aunque estos no están dentro de los criterios diagnósticos, nosotros pensamos que si se deben considerar como elementos auxiliares para la detección precoz de la enfermedad. Está pendiente definir su valor en el algoritmo diagnóstico.

Sabemos que la aplicación de un protocolo de RM que considera a las articulaciones sacroilíacas y columna vertebral está cambiando la forma de ver a las espondiloartritis en el mundo y esperamos que su aplicación también se extienda a nuestro país.

\section{Referencias}

1. Kellgren JH. Diagnostic criteria for population studies. Bull Rheum Dis 1962; 13: 291-2.

2. Linden S Van Der, Valkenburg HA, Cats A. Evaluation of Diagnostic Criteria for Ankylosing Spondylitis. Arthritis Rheum 1984; 27 (4): 361-8.

3. Amor B, Dougados M, Mijiyawa M. Criteria of the classification of spondylarthropathies. Rev Rhum Mal Osteoartic 1990; 57 (2): 85-9. 
4. Weber U, Maksymowych WP. Sensitivity and specificity of magnetic resonance imaging for axial spondyloarthritis. Am J Med Sci 2011; 341 (4): 272-7.

5. Oostveen J, Prevo R, den Boer J, van de Laar M. Early detection of sacroiliitis on magnetic resonance imaging and subsequent development of sacroiliitis on plain radiography. A prospective, longitudinal study. J Rheumatol 1999; 26 (9): 1953-8.

6. Rudwaleit $M$, van der Heijde $D$, Landewé R, Listing J, Akkoc N, Brandt J, et al. The development of Assessment of SpondyloArthritis international Society classification criteria for axial spondyloarthritis (part II): validation and final selection. Ann Rheum Dis 2009; 68 (6): 777-83.

7. Bennett P, Wood P. Population studies of the rheumatic diseases. Amsterdam, Netherlands Excerpta Medica Found 1968; 456-7.

8. Landewé RBM, Hermann K-GA, van der Heijde DMFM, Baraliakos X, Jurik A-G, Lambert RG, et al. Scoring sacroiliac joints by magnetic resonance imaging. A multiple-reader reliability experiment. J Rheumatol 2005; 32 (10): 2050-5.

9. Maksymowych WP, Inman RD, Salonen D, Dhillon SS, Williams M, Stone M, et al. Spondyloarthritis research Consortium of Canada magnetic resonance imaging index for assessment of sacroiliac joint inflammation in ankylosing spondylitis. Arthritis Rheum 2005; 53 (5): 703-9.

10. Lukas C, Braun J, van der Heijde D, Hermann K-GA, Rudwaleit M, Østergaard M, et al. Scoring inflammatory activity of the spine by magnetic resonance imaging in ankylosing spondylitis: a multireader experiment. J Rheumatol 2007; 34 (4): 862-70.

11. Rudwaleit M, Schwarzlose S, Hilgert ES, Listing J, Braun J, Sieper J. MRI in predicting a major clinical response to anti-tumour necrosis factor treatment in ankylosing spondylitis. Ann Rheum Dis 2008; 67 (9): 1276-81.

12. Baraliakos X, Landewé R, Hermann K-G, Listing J, Golder W, Brandt J, et al. Inflammation in ankylosing spondylitis: a systematic description of the extent and frequency of acute spinal changes using magnetic resonance imaging. Ann Rheum Dis 2005; 64 (5): 730-4.

13. Althoff CE, Feist E, Burova E, Eshed I, Bollow M, Hamm
B, et al. Magnetic resonance imaging of active sacroiliitis: do we really need gadolinium? Eur J Radiol 2009; 71 (2): 232-6.

14. Hermann KA, Landewé RBM, Braun J, Heijde DMFM Van Der. Magnetic Resonance Imaging of Inflammatory Lesions in the Spine in Ankylosing Spondylitis Clinical Trials : Is Paramagnetic Contrast Medium Necessary? J Rheumatol 2005; 32 (10): 2056-60.

15. Weber U, Pedersen SJ, Østergaard M, Rufibach K, Lambert RGW, Maksymowych WP. Can erosions on MRI of the sacroiliac joints be reliably detected in patients with ankylosing spondylitis? - A cross-sectional study. Arthritis Res Ther 2012; 14 (3): R124.

16. Rudwaleit M, Jurik AG, Hermann KA, Landewé R, van der Heijde D, Baraliakos X, et al. Defining active sacroiliitis on magnetic resonance imaging (MRI) for classification of axial spondyloarthritis: a consensual approach by the ASAS/OMERACT MRI group. Ann Rheum Dis 2009; 68 (10): 1520-7.

17. Levine DS, Forbat SM, Saifuddin A. MRI of the axial skeletal manifestations of ankylosing spondylitis. Clin Radiol 2004; 59 (5): 400-13.

18. Bennett a N, Rehman A, Hensor EM, Marzo-Ortega H, Emery P, McGonagle D. Evaluation of the diagnostic utility of spinal magnetic resonance imaging in axial spondylarthritis. Arthritis Rheum 2009; 60 (5): 133141.

19. Bennett AN, Marzo-Ortega H, Rehman A, Emery P, McGonagle D. The evidence for whole-spine MRI in the assessment of axial spondyloarthropathy. Rheumatology (Oxford) 2010; 49 (3): 426-32.

20. Maksymowych WP, Chiowchanwisawakit P, Clare T, Pedersen SJ, Østergaard M, Lambert RGW. Inflammatory lesions of the spine on magnetic resonance imaging predict the development of new syndesmophytes in ankylosing spondylitis: evidence of a relationship between inflammation and new bone formation. Arthritis Rheum 2009; 60 (1): 93-102.

21. Chiowchanwisawakit P, Lambert RGW, Conner-Spady B, Maksymowych WP. Focal fat lesions at vertebral corners on magnetic resonance imaging predict the development of new syndesmophytes in ankylosing spondylitis. Arthritis Rheum 2011; 63 (8): 2215-25. 